\title{
Automatic Curve Extraction for Digitizing Rainfall Strip Charts
}

\author{
H. E. van Piggelen, T. Brandsma, And H. Manders \\ Royal Netherlands Meteorological Institute, De Bilt, Netherlands \\ J. F. LICHTENAUER \\ Operator Group Delft, Delft, Netherlands
}

(Manuscript received 11 June 2010, in final form 20 January 2011)

\begin{abstract}
A method has been developed that largely automates the labor-intensive extraction work for large amounts of rainfall strip charts and paper rolls. The method consists of the following five basic steps: 1) scanning the charts and rolls to high-resolution digital images, 2) manually and visually registering relevant meta information from charts and rolls and preprocessing rolls to locate day transitions, 3) applying automatic curve extraction software in a batch process to determine the coordinates of cumulative rainfall lines on the images, 4) postprocessing the curves that were not correctly determined in step 3, and 5) aggregating the cumulative rainfall in pixel coordinates to the desired time resolution. The core of the method is in step 3. Here a color detection procedure is introduced that automatically separates the background of the charts and rolls from the grid and subsequently the rainfall curve. The rainfall curve is detected by minimization of a cost function. In total, 321 station years of locations in the Netherlands have successfully been digitized and transformed to long-term rainfall time series with 5-min resolution. In about $30 \%$ of the cases, semiautomatic postprocessing of the results was needed using a purpose-built graphical interface application. This percentage, however, strongly depends on the quality of the recorded curves and the charts and rolls. Although developed for rainfall, the method can be applied to other elements as well.
\end{abstract}

\section{Introduction}

The archives of meteorological and hydrological services around the world contain large amounts of strip charts and paper rolls. These charts and rolls often comprise information that are not yet available in digital form but are of interest for climate research and applications.

Strip charts and paper rolls have been used for the continuous recording of parameters like air temperature, humidity, rainfall intensity, wind speed and direction, and air pressure. In many parts of the world the recorders have now been replaced by electronic measuring devices whose output is digitally stored in databases. The time resolution of the latter is often an order of magnitude larger than that for the data that have been manually extracted from the strip charts and paper rolls.

Corresponding author address: Theo Brandsma, Wilhelminalaan 10, Royal Netherlands Meteorological Institute, 3732 GK De Bilt, Netherlands.

E-mail: theo.brandsma@knmi.nl
For instance, in the Netherlands temperature and rainfall are now operationally stored at 10 -min resolution, and even at 1-min resolution for some stations, while the strip charts have been used mostly for extracting hourly to 3-hourly values. Nevertheless, the charts and rolls can still be used to extract information with a time resolution of about 5-10 min. To do this manually for many years of data is labor intensive, and therefore it is often not feasible.

Here we present a framework that automates the labor-intensive extraction work for large amounts of rainfall strip charts and paper rolls. The resulting long time series of high-resolution rainfall series are needed for several purposes. First, they are the basis for the calculation of rainfall intensity-duration-frequency curves and design-storm estimation in hydrological analysis and engineering design (Di Baldassarre et al. 2006; Madsen et al. 2002; Alila 2000). These types of analyses mainly use the annual maximum amounts of the rainfall time series for durations ranging between about $5 \mathrm{~min}$ and 1 day. Second, in contrast to the latter, complete high-resolution series are used as input, for example, for urban drainage 
simulation (Mikkelsen et al. 2005). Third, high-resolution rainfall series are increasingly being used for the validation of climate models (May 2007; Lenderink and Meijgaard 2008) and for the calibration of stochastic rainfall models (Leonard et al. 2008; Burton et al. 2008). Finally, these rainfall series may also be used for climate change detection (Klein Tank and Können 2003; Klein Tank et al. 2006).

Several commercial and free software packages are available for tracing lines. However, these packages are meant to trace one line at a time and require manual navigation, such as indicating the position of the axes, indicating the beginning and the end of the curve to be traced, and specifying the color of that curve. The packages have difficulties handling background grids and curved axes, which are common for meteorological strip charts. Consequently, they are not suitable for processing large amounts of strip charts.

The development of automatic curve extraction (ACE) software that enables the processing of large amounts of strip charts is still in its initial phase. Deidda et al. (2007) developed an automatic system for signal recognition from tipping-bucket gauge strip charts. Their procedure requires the specification of a color component segmentation threshold, the plot line thickness, the magnitude of the vertical excursion corresponding to a gauge tip, and some other instrument-specific parameters. In this paper we extend and largely automate the approach of Deidda et al. (2007) for continuous rainfall curves belonging to the siphoning-type rain gauges, which are common in many parts of the world. Although the framework is developed for rainfall, it can be suited for other elements as well. Figure 1 shows the following five basic steps of the framework: 1) image scanning, where the strip charts or paper rolls are scanned to digital images; 2) preprocessing, consisting of metadata extraction and the definition of day transitions on the roll images; 3 ) application of the ACE method to determine the coordinates of the lines on the images (all steps within the dashed box); 4) result postprocessing with a graphical user interface (GUI) to visually inspect the results of the curve extraction method and to correct possible errors resulting from step 3; and 5) result aggregation, which translates the coordinates of cumulative rainfall curves to time series with the desired time resolution.

We have applied the method to 321 station years of rainfall strip charts and paper rolls, resulting in 321 stations years of 5-min-resolution rainfall time series.

In the remainder of the paper we first describe the data in section 2. The several steps included in Fig. 1 are described in section 3. In section 4 we present some of the results. Section 5 gives background information on the software developed for this work. Finally, in section

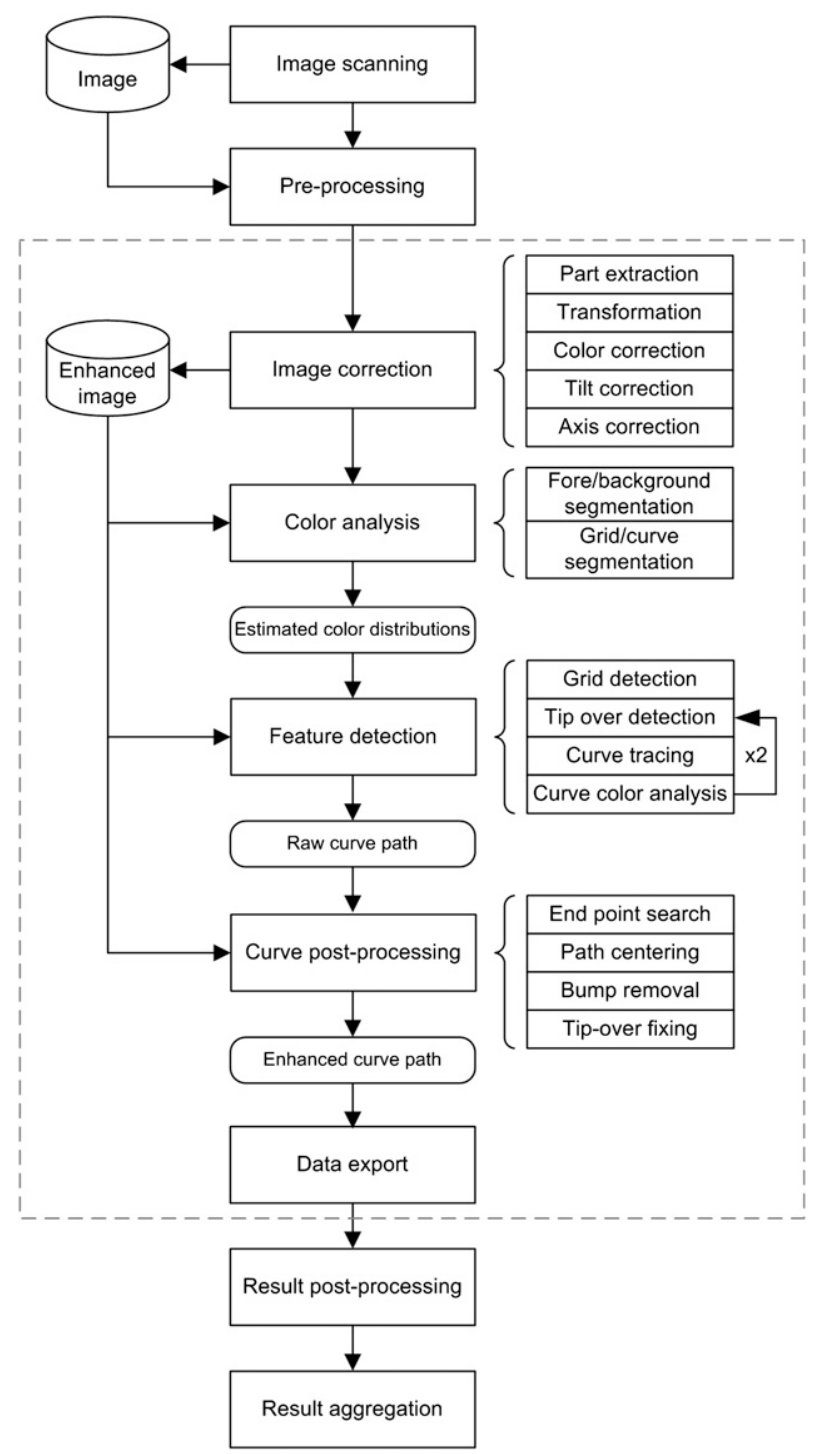

FIG. 1. Schematic overview of the automatic curve extraction framework. The part within the dashed box is fully automatic. See text for details.

6 we discuss the results, followed by some conclusions in section 7 .

\section{Data}

The total number of daily rainfall strip charts in our archive is about 110000 and the number of rolls is about 5000. A typical strip chart size is $40 \mathrm{~cm} \times 8 \mathrm{~cm}$. Each roll contains, on average, 15 days and can be up to $40 \mathrm{~m}$ in length with heights varying between 14 and $29 \mathrm{~cm}$. The length of a single day on a roll is about $1.5 \mathrm{~m}$.

A typical strip chart is shown in Fig. 2. In this figure, several features of a chart can be distinguished: on the 

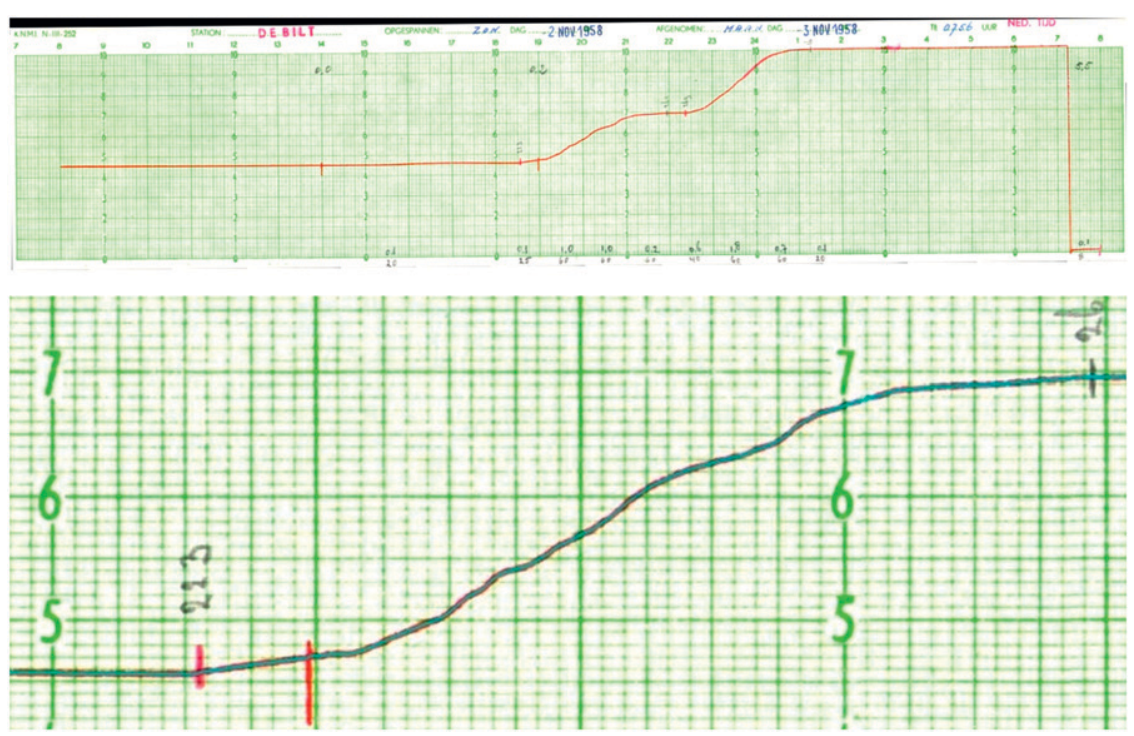

FIG. 2. Example image of (top) an unprocessed scanned original strip chart of station De Bilt and (bottom) the corresponding trace result (cutout). The original image is slightly tilted, clarified by the black fill on top of it, but is already in the correct processing orientation. (bottom) The tilt has been corrected and the result of the automatic tracing algorithm is illustrated (semitransparent blue line). Time markings made by an observer are shown (small red vertical lines). A few handwritten annotations are also visible (black).

paper-colored background a green-colored grid is printed with a red-colored curve on top of it representing the cumulative rainfall amount. On top of the chart, the name of the station (De Bilt) and two dates are stamped. The first date represents the date the strip was positioned into the pluviograph; the second corresponds to the date the strip was taken off. The exact times are usually handwritten alongside the stamps. In general, the times are fixed at 0800 UTC, but variations of some minutes sometimes occur. Note that the start and end times of the recording are not necessarily equal to the grid start and end times. Also visible are some markings written down by an observer or analyst after the recording took place.

On the far right of the strip, a so-called tip-over moment is visible as a nearly vertical line drawn in the red curve color. This line corresponds to the time when the pluviograph automatically emptied its reservoir (here when the reservoir contains $10 \mathrm{~mm}$ of rainfall). In rare cases, the emptying was done by the observer. The tipover moments are a common feature and need to be detected in order to successfully extract the rainfall data.

In contrast to charts, rolls have no fixed times for placement and removal. Similar stamps or annotations of measurement location are mostly visible only at the beginning or end of the roll together with a written date and the recording times. The basis information on rolls is identical to that on charts.
There is large variation in the layout of both strips and rolls. The most notable variation is in the colors used for the grid and the curve on the strip charts (Table 1). The grid color is most often green, but in early years red and black were also applied. Red and blue are the most commonly used curve colors. In rare cases $(0.2 \%)$, the grid and curve have almost the same red color and are therefore hardly distinguishable. This complicates the detection process. The background (paper) color is almost always close to white. However, on very old charts this has darkened to a yellow-brownish color.

Regarding the grid and curve, the rolls have a similar appearance to the charts, with the exception that the grid is oriented vertically instead of horizontally. The color scheme only varies slightly. The grid is mostly dark

TABLE 1. Frequency of occurrence of grid and curve color combinations and their totals for all examined charts. The green grid-red curve color combination is most common.

\begin{tabular}{llllllr}
\hline & & \multicolumn{4}{c}{ Curve color (\%) } & \\
\cline { 3 - 6 } & & Black & Purple & Blue & Red & Total \\
\hline Grid color (\%) & Black & 0 & 0 & 0 & 1.0 & 1.0 \\
& Orange & 0 & 8.4 & 0.4 & 0 & 8.8 \\
& Brown & 0.1 & 0.1 & 0 & 0.5 & 0.7 \\
& Green & 0 & 0 & 1.2 & 58.2 & 59.4 \\
& Gray & 0 & 3.6 & 0 & 8.6 & 12.2 \\
& Red & 2.6 & 13.9 & 1.2 & 0.2 & 17.9 \\
& Total & 2.7 & 26.0 & 2.8 & 68.5 & 100.0 \\
\hline
\end{tabular}


gray colored on a light gray background with a redcolored rainfall curve on top of it. On the roll images, the day transitions correspond exactly to the 24-h markings that are visible on the graph. In some cases, time markings are visible that are manually drawn by an observer. These time markings can be of any time and can be located anywhere on the roll independent of the underlying grid. Furthermore, in some cases, a roll contains two separate grids, for example, for recording another meteorological variable. Therefore, the upper and lower boundary of the correct grid must be known as well. Because for rolls the exact positions of the grid boundaries and the type and position of markers are not known in advance, manual preprocessing is necessary (see section 3 ).

A variation that appears for strip charts only is the presence of curved vertical axes. This occurs for the socalled balance-type pluviographs that were occasionally used in the first part of the twentieth century. The graph start and end times may also vary through the years.

A metadatabase was constructed defining for each measurement location the periods with identical features. The start and end times of the graph and the presence of a curved axis are derived from this database.

\section{Methods}

In this section we describe the details of the several steps in Fig. 1. In section 3a we first describe the pre- and postprocessing steps, including the image scanning and results aggregation. Thereafter, in section $3 \mathrm{~b}$, we describe the core of the framework: the ACE as enclosed in the dashed box in Fig. 1.

For ease of exposition, most of the time, strip charts are used as reference. However, the methodology is equally applicable for the roll images with the exception of the grid detection step. The latter is dealt with in section $3 b$.

\section{a. Pre- and postprocessing}

\section{1) SCANNING STRIP CHARTS AND ROLLS}

Strip charts were scanned using the Canon DR5010C transit scanner, which is capable of scanning at a speed of 20 charts per minute at a maximum resolution of 600 dots per inch (dpi). Because physically cutting the rolls was not allowed, the Contex Chameleon G600 scanner, designed for large image sizes, was used to scan the rolls with a speed of $150 \mathrm{~cm} \mathrm{~min}{ }^{-1}$ at 300-dpi resolution. Using these resolutions, a number of $10-15$ pixels per curve and grid line thicknesses is achieved, which is considered sufficient. The curves can now easily be distinguished from the background and the center of the line can be accurately estimated.
Because of the large number of rainfall strips charts and rolls, file storage requirements are a concern. Together with the output data resulting from the framework, the necessary amount of data to be stored may become rather large (in our case, over $3 \mathrm{~TB}$ ). To reduce the amount of storage space needed, the following specific scanning options have been applied. The strip charts were stored as 24-bit color jpeg images and the rolls were stored as standard Windows Bitmap (bmp) images using an adapted 256-color palette. Although the JPEG compression scheme for the charts is specifically designed for photographic images, the resulting image quality is still sufficient for effective segmentation and detection.

\section{2) PRePRocessing}

For each measurement location, periods with identical features (e.g., the start and end times of the graphs and the presence of a curved vertical axis) have to be identified. This information is stored in a metadatabase and used later on during the processing of the images.

A property of the rolls is that they mostly contain multiple-day recordings in a sequence. For a correct detection of the rainfall values on the rolls, the exact start and end date and the time of the roll recording are needed, as well as the exact locations of the day transitions and the upper and lower grid boundaries. Sometimes, only arbitrary time markings made by the observer are available. Before the application of ACE, the roll images were digitally cut to make sure that each day on these images is processed correctly. To avoid the errorprone automatic detection of these lines and markings, the day transitions and grid boundary positions were identified manually. To facilitate this work, we developed a special application, called Roller, which was capable of handling the large bitmap images and providing the necessary tools to accomplish the marking of the marker and grid position locations.

\section{3) Semiautomatic postprocessing}

The results of ACE are examined using PostACE (illustrated in section $3 b$ ). We developed this software to visually inspect the results of the automatic extraction and to adjust the found features in the images if needed. If necessary, the extraction with ACE can be repeated within PostACE using user-defined image features, such as altered tip-over moment positions.

In case the trace still does not match the exact position of the curve found on the image, correction lines can be drawn to force the vertical position of the curve points across a specified interval. In addition, polygons can be drawn to exclude the enclosed pixels during the curve tracing phase by setting the corresponding probability values to zero (background). 


\section{4) Result aggregation}

Finally, the extraction results of the individual images are combined into a rainfall intensity table with arbitrary time resolution and time range. Here, the time resolution was set to $5 \mathrm{~min}$, corresponding to a width of roughly 30 pixels for the strip charts and 60 pixels for rolls. Using the start and end points of each curve with corresponding times, the exact pixel positions of the 5-min intervals are determined so that each interval exactly matches one of the 12 intervals in an hour. The actual difference in pixel height within each interval corresponds to the rainfall intensity during that period. In addition, the resulting hourly and daily precipitation values are compared to previously known values to check if the found values are correct. If values are too far off, then (manual) reprocessing can be considered.

\section{b. $A C E$}

\section{1) IMAGE CORRECTION}

Image correction for rolls starts with the extraction of the correct part of the scanned image, because only a single day is processed at a time. Images are transformed automatically to the common chart orientation as shown in Fig. 2 (time running from left to right and cumulative rainfall increasing from bottom to top). Note that this does not include tilt correction. Optionally, color correction can be applied if images do not have enough contrast to successfully complete the detection process.

Each strip or roll is scanned in a machine that automatically transports it, along its length, over a line-scan sensor. Such an automatic scan-feeding process allows for the efficient processing of large amounts of strips and long rolls. Unfortunately, small displacements of the paper inevitably occur during the scanning process. The effect of these displacements is a geometrical transformation of the charts in the recorded images (the upper image in Fig. 2 shows an exaggerated example). This transformation can best be described as a local shearing of the chart: vertical lines remain mostly vertical, while horizontal lines can become slanted, or even curved. In the remainder of this article, we will refer to this nonlinear distortion as tilt. Because the unpredictable nature of tilt deformation does not allow straightforward modeling as a global geometric transformation, we have chosen to correct for it at the local level of image columns.

Vertical realignment of all image columns is achieved by applying tilt correction. This process utilizes the repetitiveness of the recurring horizontal lines of the grid in vertical direction to estimate the necessary local shift.
By correlating the pixel columns of the image with a centrally located reference column, the shift for that part compared to the central part of the image can be estimated. To speed up the comparison, only one of every 200 pixel columns is compared. Only pixel columns that cross clear horizontal grid lines and are not on top of vertical grid lines or image artifacts, are considered suitable and are taken into account. This is determined by calculating the autocorrelation of a column. If no suitable column is found at a certain location, neighboring columns within a range of 75 pixels on both sides are checked for suitability. Considering the initial 200 pixel interval, ultimately a shift is measured within every 50-350 pixels, ensuring a proper estimate of the image tilt. Once the shifts have been found, the set of shifts is filtered so that any erroneous peak shifts are removed. Finally, the shifts are linearly interpolated and applied to each pixel column of the image. In general, a smooth tilt correction is realized, but in rare cases a more complex operation is required to correct for tilt. To avoid such an error-prone and time-consuming imagewarping operation, these corresponding strip charts have been rescanned.

Curved vertical axis correction involves similar calculations. Instead of columns, all pixel rows are cross correlated with a suitable reference row located centrally in the image. The curve of the vertical axis can be described by a second-degree polynomial. We fitted shifts to this function resulting in a smooth correction. This step is only performed if the metadatabase indicates curvature of the vertical axis.

\section{2) ForEGROUND-BACKGROUND SEGMENTATION}

The color distributions of grid and paper are robustly estimated in a two-step clustering process, guided by the following four assumptions about relative coverage and intensity of blank paper and grid lines:

1) blank paper covers about $90 \%$ of the surface between grid lines,

2) blank paper is brighter than anything else in the image,

3) grid lines cover about $10 \%$ of the surface within a grid area, and

4) grid lines are darker than anything else in the image.

Assumptions 1 and 3 are not strict. ACE should be able to handle a reasonable amount of deviation from the expected coverage. Assumption 2 holds for all of our examples, because adding ink to paper usually leads to a reduction of brightness. However, assumption 4 only holds when an image contains nothing else but grid and paper. It is often violated when a piece of curve, handwritten text, or markers are present as well. This is why 
we have split the color estimation process into a cascade of two clustering procedures. We first estimate grid and paper color in small grid blocks (SGBs) of the image, and, in the second step, apply clustering to the outcomes of all SGBs. This results from a (crucial) fifth assumption:

5) The grid area of an image can be broken up into square blocks, of which more than $50 \%$ only contain grid and paper, without a curve, markings, or handwriting.

Assumption 5 means that assumptions $1-4$ hold for at least $50 \%$ of the SGBs, resulting in a reliable estimation of paper and grid color. For the remaining SGBs, successful color estimation cannot be guaranteed. By subsequently clustering the estimated mean paper and grid colors of all SGBs, the failed SGB outcomes are discarded and successful results can be combined to increase estimation accuracy. Figure 3 shows a schematic overview of the color modeling process. The following paragraphs describe the process in detail.

\section{(i) Clust1: SGB color modeling}

The size of SGBs is chosen to be greater than or equal to one period (the pixel interval between the thick grid lines) of the grid pattern (both horizontally and vertically), so that the thick grid lines are always within such an SGB. Larger blocks would not be beneficial, because they would result in a higher percentage of blocks containing a piece of the curve or other ink from stamps or handwriting (possibly violating assumption 5). The SGBs are only extracted from the central region of the image, to ensure that they actually contain grid lines. It is possible to extract overlapping blocks from an image in order to maximize the percentage of blocks that do not contain anything but blank paper and grid. However, this results in more blocks, and thus a higher computational load. For our data the use of adjoining blocks was sufficient.

The first step of clustering is applied to every individual SGB. The clustering procedure is a modification of expectation maximization (Hogg et al. 2005). In a few iterations (five were enough to converge to a stable result), the estimates of the mean and covariance of the grid and paper color are updated by recomputing the mean color of each cluster. A good initialization of the paper and grid color is crucial for a successful clustering. The initialization of paper color $c_{P}^{(0)}$ is done by computing the mean color $\bar{c}(B)$, where $B$ denotes the set of the brightest $90 \%$ of pixels in the SGB. Similarly, the initial grid color $c_{G}^{(0)}$ is found by the mean color $\bar{c}(D)$, where $D$ denotes the set of the darkest $10 \%$ of pixels in the SGB. Brightness $I$ of a pixel $x$ is defined as

$$
I(x)=\frac{r(x)+2 g(x)+b(x)}{4},
$$

where $r(x), g(x)$, and $b(x)$ are the red, green, and blue components of $x$, respectively. This is because multiplications and divisions by two are executed much faster in digital hardware, and most of current imaging devices use a ratio of two green sensors for every pair of blue and red sensors. This means that green is more reliably measured than either red or blue. The mean color $\bar{c}(S)=\left[\bar{r}\left(x_{i}\right), \bar{g}\left(x_{i}\right), \bar{b}\left(x_{i}\right)\right]^{\mathrm{T}}$ of the colors $c\left(x_{i}\right)=\left[r\left(x_{i}\right)\right.$, $\left.g\left(x_{i}\right), b\left(x_{i}\right)\right]^{\mathrm{T}}, i \in S$ of the pixels in set $S$ is defined as

$$
\bar{c}(S)=\frac{1}{N} \sum_{i \in S} c\left(x_{i}\right)
$$

where $N$ is the size of set $S$. Each iteration $k$ starts with regrouping SGB pixels to the paper $P^{(k)}$ and the grid $G^{(k)}$ clusters. This is based on the Euclidean distance to the respective estimates of mean colors in RGB space. To be robust against deviations from the expected coverage, only the closest half of the respective expected coverage is used. This means that the paper cluster $P^{(k)}$ is formed by the $45 \%$ closest SGB pixels to $c_{P}^{(k-1)}$, while the grid cluster $G^{(k)}$ is formed by the $5 \%$ closest SGB pixels to $c_{G}^{(k-1)}$. Finally, the colors are re-estimated by $c_{P}^{(k)}=\bar{c}\left[P^{(k)}\right]$ and $c_{G}^{(k)}=\bar{c}\left[G^{(k)}\right]$.

Both $c_{P}^{(k)}$ and $c_{G}^{(k)}$ of the last iteration are used as the estimated paper $c_{p}(m)$ and grid $c_{G}(m)$ color, respectively, for the SGB with index $m$. The $3 \times 3$ covariance matrix of the grid color in red-green-blue (RGB) space $\Sigma_{G}(m)$ is estimated over the last grid cluster $G_{1}^{(k)}$. In the scanned images, the background is often saturated. This makes it impossible to calculate the covariance of paper color $\Sigma_{P}(m)$. However, most of the variation in paper and grid color is caused by a gradual transition between grid and paper. These smooth transitions result from 1) variations in the amount of grid ink on the chart-rolls, and 2) interpolation around the edges during the scanning process. Consequently, the covariances of paper and grid are quite similar and can be assumed to be the same.

\section{(ii) Clust2: Combining SGB outcomes}

From the procedure described above, we now have two sets of color values $c_{p}(m)$ and $c_{G}(m), m \in\{1, \ldots$, $\left.N_{\mathrm{SGB}}\right\}$, where $N_{\mathrm{SGB}}$ is the total number of SGBs. A single-color clustering is applied to both sets, which differs from the above procedure for the individual SGBs only in the computation of the initial color and the percentage of the closest values assigned to a cluster. The initial color for each set (paper and grid procedures are now identical) is estimated as the mean 


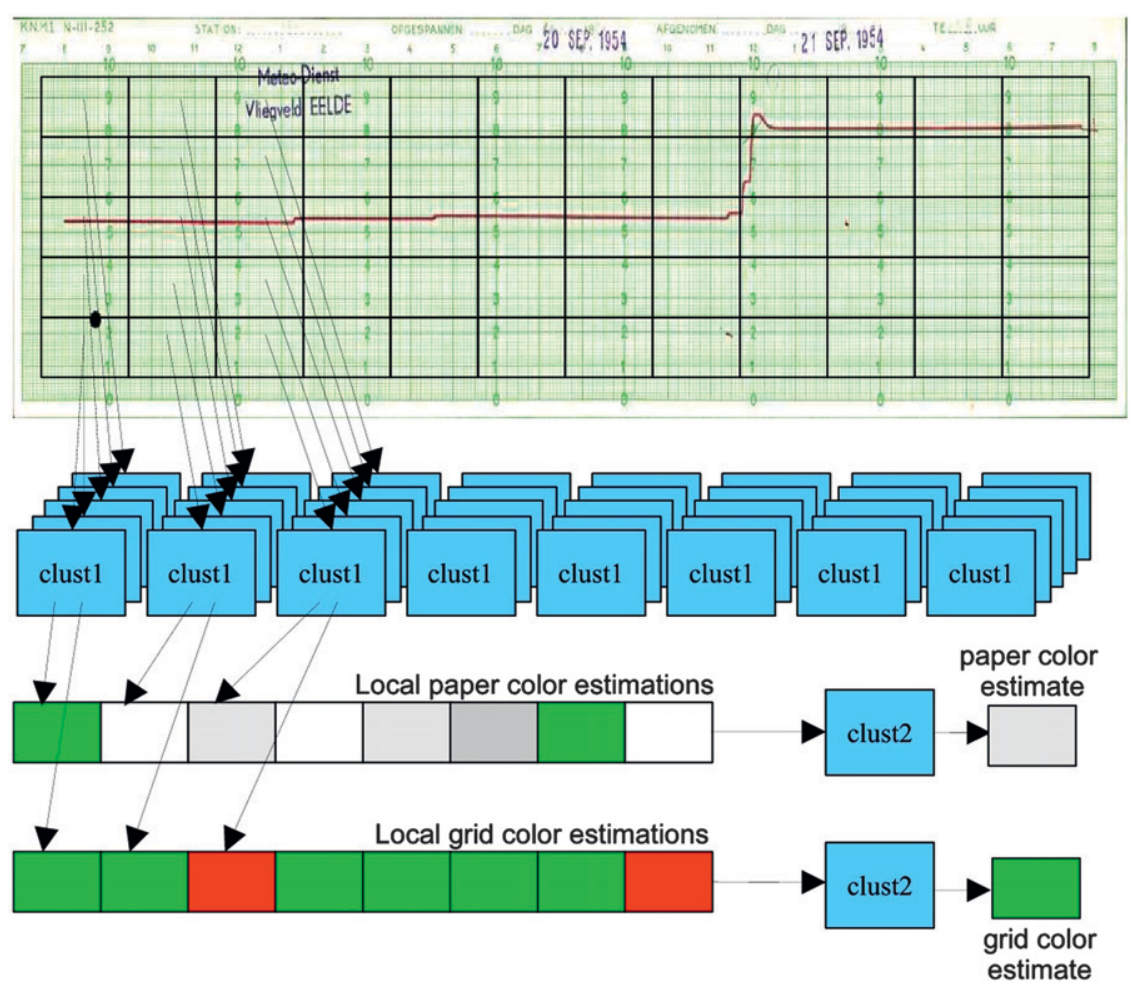

FIG. 3. Schematic overview of the automatic color modeling process. The strip chart is divided into blocks placed centrally on the image. Of each block, the grid and paper pixels are clustered into two segmentation groups (clust1) of which the calculated mean colors are respectively shown in the two horizontal sequences of colored rectangles. (top) Estimations of the average color of the blank paper. For some blocks, the clustering may have gone wrong, ending up with green grid pixels falsely classified as blank paper. (bottom) Some clusterings can converge to falsely classify the red pixels of the curve as grid. The second clustering step (clust2) selects the $50 \%$ of local color estimations that are found most likely to be correct, discarding the results of failed local clusterings. The global grid and paper colors are derived from these selected groups. See text for a detailed explanation of each step.

color of the entire set. The $50 \%$ closest values are now used for assigning values $c_{p}(m)$ and $c_{G}(m)$ to clusters $P_{2}^{(k)}$ and $G_{2}^{(k)}$, respectively, in clustering iteration $k$. After the final iteration, the SGB grid covariance matrices $\Sigma_{G}(m), m \in G_{2}^{(k)}$ are averaged per matrix element to obtain an overall estimate of the grid color covariance $\Sigma_{G}$.

\section{(iii) Grid likelihood}

For the estimation of grid borders, it is important to have an estimate of the grid pattern. The likelihood of a pixel $x$ belonging to a grid line is estimated by

$$
L_{G}(x)=255\left\{1-\min \left[1, \frac{\left\|c(x)-c_{G}\right\|}{\left\|c_{P}-c_{G}\right\|}\right]\right\}
$$

where $\|\cdot\|$ denotes the L2 norm of a vector (Euclidean distance in RGB space) and $\min (a, b)$ equals $a$ for $a<$ $b$ and $b$ otherwise. The normalization with the distance between the paper and grid color ensures that paper color is given a likelihood around 0 , while the multiplication with 255 makes the results of a whole image suitable to be saved as a typical 8-bit monochrome image. Because this measure does not correspond to actual statistics, it represents a fuzzy classification rather than likelihood. The upper image in Fig. 4 gives an example of a grid likelihood image.

\section{(iv) Foreground likelihood}

Now that we have estimated the statistics of paper and grid color, we can use this information to recognize anything that looks like neither of them. In the remainder of this article, we will refer to background as denoting either the paper or grid that is not covered by ink or other local discolorings. With foreground we refer to any type of discoloring of the paper other than the grid, such as the measurement curve, handwriting, stamps, or stains. 

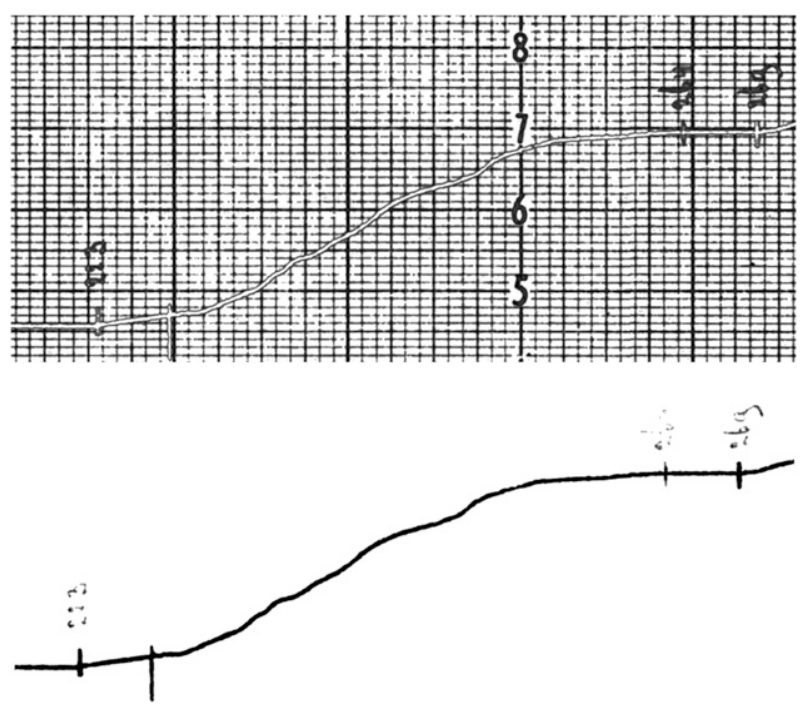

FIG. 4. A grid and curve likelihood generated from the same part of the same source image. (top) The grid likelihood image is depicted resulting from the fuzzy classification segmentation using the background color model as input. The darker the colors, the more likely the pixels belong to the grid. Grid lines are clearly visible while the curve is only slightly distinguishable. (bottom) The curve likelihood image is shown resulting from the fuzzy classification segmentation using the curve color model as input. As a result, grid and background intensity are suppressed making the curve easily traceable.

The Mahalanobis distance is used to take into account the directional dependency of the variance of background color in RGB space. Simply combining the Mahalanobis distances to mean paper $c_{P}$ and grid color $c_{G}$ will lead to the classification of the smooth transitions between the grid and paper as foreground. This is because pixels on the smooth edges are unlike grid color, but also unlike paper color. Instead, we use the knowledge that the colors on these soft edges are a linear combination of grid and paper color (assuming the smoothing is the same in all color channels). Instead of computing the Mahalanobis distance to $c_{P}$ and $c_{G}$, a reference background color $c_{G P}(x)$ is defined as the projection of a pixel onto the vector $\mathbf{v}_{G P}$ between $c_{G}$ and $c_{P}$,

$$
\begin{aligned}
c_{G P}(x) & =c_{G}+\mathbf{v}_{G P} \cdot \max \left\{0,\left[c(x)-c_{G}\right]^{\mathrm{T}} \mathbf{v}_{G P}\right\} \\
|\mathbf{v}|_{G P} & =\frac{c_{P}-c_{G}}{\left\|c_{P}-c_{G}\right\|},
\end{aligned}
$$

where $\max (a, b)$ is $a$ for $a>b$ and $b$ otherwise. The max function ensures that when the projection falls below $c_{G}$ (darker than grid color), $c_{G}$ is used directly as the background color. Now, the unnormalized foreground likelihood $L_{\mathrm{FG}}^{*}$ is calculated as
$L_{\mathrm{FG}}^{*}(x)=\frac{\sqrt{\left[c(x)-c_{G P}(x)\right]^{\mathrm{T}} \Sigma_{G}^{-1}\left[c(x)-c_{G P}(x)\right]}}{I(x)}$.

Small values of $L_{\mathrm{FG}}^{*}$ are more likely related to background pixels, while high values are more likely related to foreground pixels. The inverse scaling of $L_{\mathrm{FG}}^{*}$ by brightness $I(x)$ results in more tolerance to background discoloring in the brighter background colors (which belong to the blank paper). This improves the foreground likelihood estimate when variation of the color of paper is higher than variation in the grid color. Two sources of image color variation apply more to the paper than to the grid. First, long-term exposure of paper to either oxygen or moisture changes its physical color. Such exposure may vary across the surface area, causing variation in the paper color. Second, the brightest paper regions may become (partially) saturated during the automated scanning process, causing a shift in the estimated paper color $c_{P}$ from the actual color value (which lies outside the limits of the RGB representation). The inverse scaling with $I(x)$ takes into account the extra uncertainty originating from both causes of color variation. The difference between this model and a straightforward Mahalanobis distance is shown in Fig. 5. In Fig. 5a an elliptical surface in RGB space is plotted that has an equal Mahalanobis distance to the mean grid color; Fig. $5 b$ shows a surface in the same space that has an equal foreground likelihood as calculated by $L_{\mathrm{FG}}^{*}(x)$.

To effectively use this foreground likelihood model, it is essential to scale it such that it yields comparable likelihood values for all images, independent of color variance or its estimation. This is done in a robust empirical estimation of the boundary value $L_{\mathrm{FG}}^{(\max B G)}$ of $L_{\mathrm{FG}}^{*}(x)$. over the SGBs that were selected for the grid color cluster $G^{(k)}$ in the last step of the clust2 procedure for grid color. For each of these SGBs, the 99th percentile of $L_{\mathrm{FG}}^{*}(x)$. is determined over all pixels in the block. A clustering of these percentiles is done that is identical to the clust2 procedure, resulting in $L_{\mathrm{FG}}^{(\max B G)}$ as the mean of the final cluster.

In addition to being scaled, the foreground likelihood is also mapped by a Gaussian function centered at 0 . This is because differences between colors with a large distance from the background colors are relatively less important. A Gaussian mapping reduces the likelihood differences between these different foreground colors, while still keeping nuances that may be important to track, such as the curve path through stains or other clutter or artifacts that are different from the estimated background colors. Because the resulting likelihood $L_{\mathrm{FG}}(x)$ does not correspond to an actual probability, it has to be regarded as a fuzzy foreground classification, 


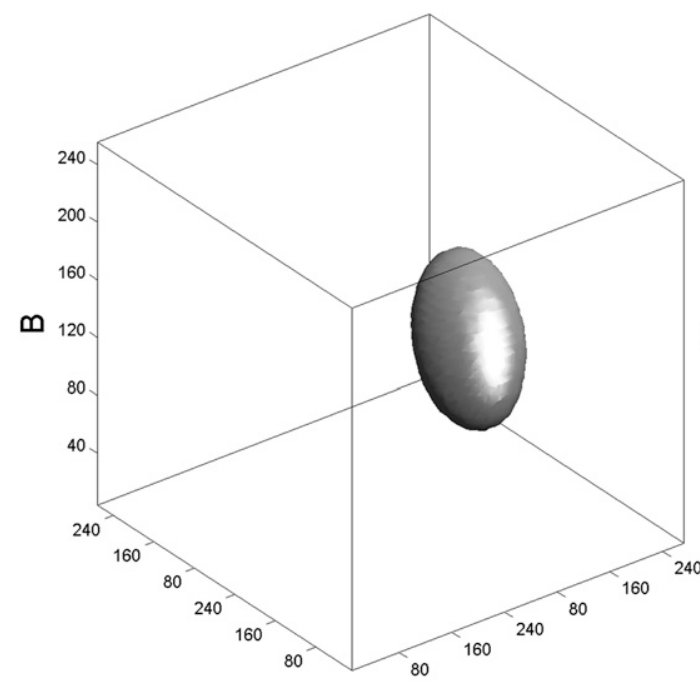

$\mathrm{R}$

\section{G}

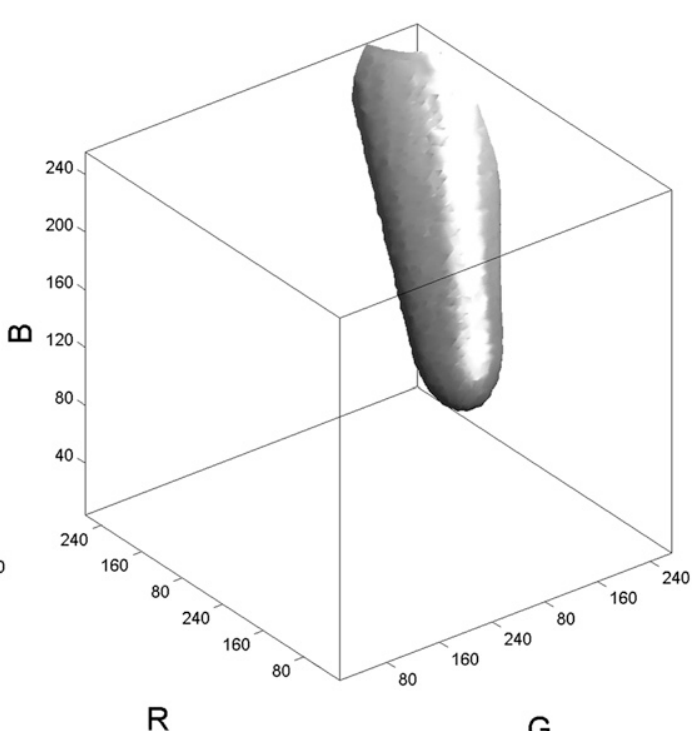

G

FIG. 5. Isosurface of likelihood in RGB space, for (a) the Mahalanobis distance to mean grid color and (b) the foreground likelihood model $L_{\mathrm{FG}}(x)$.

$$
L_{\mathrm{FG}}(x)=255\left\langle 1-\exp \left\{-\frac{1}{2}\left[\frac{\alpha_{\mathrm{BG}} L_{\mathrm{FG}}^{*}(x)}{L_{\mathrm{FG}}^{(\max \mathrm{BG})}}\right]^{2}\right\}\right\rangle,
$$

where $\alpha_{\mathrm{BG}}$ is a factor that should map most of the likelihoods in the background to the relatively flattopped region of the Gaussian function, while causing background colors to end up as much as possible in the low, flat tail. Colors for which it is difficult to distinguish whether they belong to either the background or foreground, should be mapped onto the steep edge of the Gaussian. Here $\alpha_{\mathrm{BG}}=0.4$ was used to achieve a high contrast in $L_{\mathrm{FG}}(x)$ between the foreground and background. This value was found by trail and error and was kept constant for all cases. It is for unclear charts that an appropriate nonlinear mapping of $L_{\mathrm{FG}}^{*}(x)$ may greatly increase the reliability of curve extraction. The scaling with 255 is again used for saving the result of the likelihood computations as 8-bit monochrome images. The inversed foreground likelihood $L_{\mathrm{FG}}(x)$ is used as the initial curve likelihood $L_{C}(x)=255-L_{\mathrm{FG}}(x)$ to make it suitable for the algorithm used to extract the curve. In section $3 b(7)$, an alternative curve likelihood is described that distinguishes the curve further from other foreground content. The lower image in Fig. 4 gives an example of a curve likelihood image.

\section{3) GRID DETECTION}

To transform the curve pixel coordinates into rainfall intensity as a function of time, it is necessary to know the exact locations of the grid boundaries. These represent the start and end times on the horizontal axis and the range of rainfall on the vertical axis. Using the grid likelihood image, the locations are found by estimating the mean distance between the grid lines. For each row or column the sum of likelihoods $L_{G}(x)$ [Eq. (3)] of all pixels $x$ is calculated. To make sure that the slightly slanted grid lines are incorporated in the calculation, for each row or column the sum of an imaginary slanted line with a maximum slant of 20 pixels centered on that same row or column is regarded as well. The number 20 has been found by trail and error and depends on the scanning resolution and the maximum amount of slant existing in the data. From the set of sum values the local maxima are extracted that exceed a sum value threshold of $L_{g} \in$ [0.255] times the row or column length, respectively. Initially, $L_{g}$ is set to 50 , which is safely higher than the estimated minimum value of 25.5 when only crossing perpendicular grid lines, based on an estimated ratio of grid versus background (around 0.1 times the maximum of 255). All of the maxima arising from small successive row or column variations are discarded. Now, the mean mutual distance between two successive maxima and its standard deviation indicate how well the initial distance estimation fits the image. If the standard deviation is larger than two pixels, then the estimation is probably wrong and most likely too many maxima are taken into account. Therefore, with a raised threshold $L_{g \text {,new }}=L_{g}+1$, a lower number of maxima is taken into account. With this new set of maxima the distance estimation is repeated. As soon as the estimation is accepted, the locations of the outermost maxima having 
a distance comparable with the mean are marked as the outer boundaries of the grid. If no good estimation can be found, the first maximum is used as the boundary location.

\section{4) TIP-OVER DETECTION}

Before the actual curve is traced, the tip-over moments (defined in section 2) need to be detected, because they cause the curve to be discontinuous. The detection of these tip-overs involves a similar methodology as that in the grid detection step. In the curve likelihood image, a tip-over manifests itself as an almost vertical dark line. The pixels of each column in an image are summed, and if any sudden peaks $L_{C}(x)$ are significantly lower than neighboring columns in the total set of column sums that are found, then they likely correspond to one or more tip-overs. To make sure that the more slanted tip-overs are also taken into account, an empirically estimated slant of 24 pixels regarding the whole line length is allowed. A drawback of this method is that sometimes vertical time markings made with the curve pen color are mistaken for tip-overs. During the initial tracing in the next step they will be treated as such, but during the secondary postprocessing step they will be filtered out automatically and only the actual tip-overs should remain.

Another drawback is that there is a probability that less visible tip-overs are missed during detection. These are not taken into account, preventing a correct curve trace. The manual addition of these tip-overs during postprocessing solves this issue.

In rare cases, tip-overs are retrograde (i.e., their slant is negative). The strip chart image and curve likelihood image are corrected for this artifact by moving all pixels prior to the tip-over horizontally to the left. This way, the tip-over slant is corrected and the curve tracing will not skip any parts positioned on the chart at the same time. Because this alteration is only small, the shift in time is not an issue. Prograde tip-overs (the normal situation) are not corrected because these cause no problems during tracing and slants are usually small (depending on the time needed for emptying the reservoir), and therefore they have negligible influence on the outcome.

\section{5) CURve tracing}

A dynamic programming algorithm is applied to the curve likelihood image to trace the curve on the image. The algorithm is capable of tracing a continuous curve in right-to-left direction, using the principle of optimality (Bellman 1957). It solves a globally defined problem by finding the optimal solution of the individual smaller subproblems, which together comprise this global problem. In horizontal direction, the image is divided into sections separated by the tip-over moments found in the previous step. If no tip-overs were found, then the whole image is treated as one section. For each section, the minimal cost path is determined, where the cost factors $c(x)$ are obtained from the curve likelihood values $L_{C}(x)$ at the traversed pixel locations by $c(x)=\left[255-L_{C}(x)\right]$. In a mathematical sense, the minimal cost path is determined as the sum of all the minimum cost substeps between consecutive pixel columns,

$$
\min \left[C\left(x_{1}, x_{2}, \ldots, x_{M_{\mathrm{I}}}\right)\right]=\min _{j=1, \ldots, N_{\mathrm{I}}}\left[C\left(x_{M_{\mathrm{I}}}^{j}\right)\right]
$$

with $C\left(x_{i}^{j}\right)$ the total accumulated cost at pixel $(i, j), M_{1}$ the total number of columns, and $N_{1}$ the total number of rows in the image (Sonka et al. 1998). Thus, $C\left(x_{M}^{j}\right)$ are the pixels in the last (furthest right) column containing the total sum from left to right. Starting from left, between each pair of consecutive columns the minimal cost step from a certain pixel of the right column, the node, to a pixel on the left column is determined (Fig. 6). A userdefinable change in the $y$ coordinate is allowed with this step, in our case a search width of 25 pixels, centered on the node height. Note that this implicitly limits the detectable steepness of the curve and the ultimate maximum rainfall intensity that can be determined automatically. The accumulated cost of the pixel on the left is added to the value of the pixel on the right and the corresponding optimal step (change in height) is stored. This is repeated for every column pair. In the end, each pixel in the outermost right column has the sum value of the minimal path from the left to that particular pixel on the right. Because the coordinate of the best neighboring pixel is known for each pixel, the minimum cost path can be traced back from right to left, starting from the last column pixel with the lowest total sum. Because the curve image might be noisy, the traced curve path most likely does not represent the actual path of the curve. It also runs across the entire image instead of starting and stopping at the actual curve end points. This will be dealt with later on during postprocessing.

\section{6) INITIAL CURVE POSTPROCESSING}

Because the curve now follows the exact minimal cost path, which is not necessarily the best solution, the result must be improved by repositioning the curve points to the nearby local vertical line center of the curve on the image. This line center is found by measuring the weighted sum of the intensities of the background pixels found in the row part centered to the current pixel position in the curve likelihood image. The row with the minimum background pixel intensity is most likely the vertical line center. We derived a 


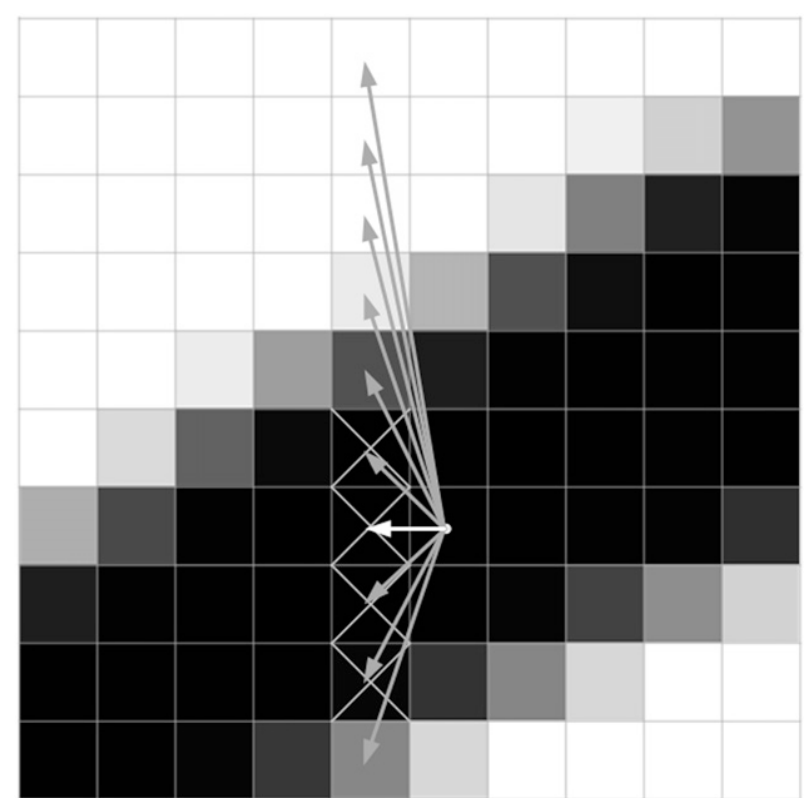

FIG. 6. Schematic representation of the principle of optimality applied to a minimal cost path problem. Visible is a matrix of pixels of which the darkest are most likely curve pixels, while the brightest are probably paper or background pixels. The intensity of the pixels represents the cost to pass through that pixel with a path. Here, the pixel with the gray dot in the middle is the actual pixel being looked at. Possible neighboring pixels are pointed to with the gray arrows. Because the minimal cost path has to be found, the best options are the central four black pixels (shaded with gray crosses). By preference, if more pixels with the lowest cost are available, then the pixel closest to the actual pixel is chosen as best neighbor (white arrow).

mathematical formula that expresses the centering of the curve point at $\left(x_{0}, y_{0}\right)$

$$
y^{\prime}=\min _{y=-N, \ldots, N}\left[\sum_{x=-M}^{M} p\left(x_{0}+x, y_{0}+y\right) w(x)\right],
$$

with $y^{\prime}$ the adjusted vertical line center, $p(x, y)$ the intensity of the pixel at coordinate $(x, y)$, and $w(x)$ a ramped weight factor for a pixel at position $x$,

$$
w(x)=1-|x / M|,
$$

where $M$ and $N$ are, respectively, the number of pixels regarded horizontally and vertically in both directions. The value of $N$ is based on the obtained line width after scanning and has been set to 10 . To successfully compare the regarded rows, the value $M$ has been set to 10 as well, which turned out to be sufficient here.

The path of the curve still traverses the whole image from left to right. For strip charts the curve itself almost always starts and ends at a different time on the grid, while for rolls it starts and ends at the left and right image borders. Therefore, the current path of the curve on the strip charts needs to be trimmed from all pixels not belonging to the rainfall curve, located outside of the beginning and end points of the curve. In this step, an initial estimate of the positions of these points is derived from the metadatabase (section 2) together with the known coordinates of the grid.

\section{7) Curve COlOR Remodeling}

The current state of the path is used to adjust the color model for the curve pixels. From all pixels belonging to the curve path, a new mean color is calculated, using a methodology that is analogous to the foreground and curve likelihood generation steps described earlier. Using the improved curve color estimate, the curve likelihood image is reconstructed again. The tip-over detection and curve tracing are repeated using the steps outlined in the previous paragraphs. In many cases this results in an improved estimate of the curve path.

\section{8) Secondary CuRVE PostPRocessing}

Secondary curve postprocessing is now applied to the newly traced curve. Again, the start and end points of the curve need to be detected for the strip charts. This time, morphological operators are applied to the path to detect the current start and end points of the curve. Because the curve likelihood image might contain gaps of undetected curve, the first path segment from the left that is longer than 150 pixels is assumed to be part of the curve line, and its outmost left point is marked as the curve start point. The same is repeated for the outermost right part. Here, the outermost right point of this segment is marked as the curve end point. Again, any path points not belonging to the curve are excluded in further calculations. This step is followed by the alteration of the path to the center of curve line, as described in the initial postprocessing step.

A common artifact is the presence of bumps in the path. These emerge because of the changes in color of the curve on the image (e.g., when running through a grid line) or because of the presence of time markings. The removal of these bumps is accomplished by convolving the traced path by a nonlinear local filter that involves morphological opening and closing operations. Figure 7 illustrates this method. The only parameter to choose here is the convolution window size, which must be larger than the largest bump present in the curve. The bump removal operation preserves the exact shape of monotonically increasing or decreasing signals, and therefore it is a safe way to improve the quality of the trace. Furthermore, a relatively large window size can be chosen for cumulative 



FIG. 7. Removing bumps from an extracted curve. The original (bumpy) curve example is repeated (gray) in all graphs. (middle) The results of a morphological closing $C\left(f_{x}\right)$ and opening $O\left(f_{x}\right)$ operation applied to the original curve $f_{x}$, respectively. (top) The intermediate results of the closing and opening operations. This shows more clearly how the results are obtained, because $C\left(f_{x}\right)=\min \max \left(f_{x}\right)$ and $O\left(f_{x}\right)=\min \max \left(f_{x}\right)$. Thus, a closing operation $C\left(f_{x}\right)$ is the result of subsequently applying a local max filter and local min filter on $f_{x}$. (bottom left) The average of the opening and closing operation that has removed the alternating bump in the middle while suppressing the two outer bumps is shown. (bottom right) These are removed by an additional subsequent application of the opening and closing operation. Reversing the order of these final opening and closing operations gives a nearly identical result.

measurements (e.g., rainfall) because the change in height of the path is not altered. At boundary events, for example, at the ends of a path or tip-over moments, we treat the line as if it would continue horizontally over the event. This means virtually replicating the last value during filtering near the events. If this would not be done, these desired extremes will also be cut off, as if they were bumps.

If there are any erroneous steps (e.g., changes in vertical height resulting from time markings) present in the path, then a correction is applied to ensure a 
continuous path. By calculating the derivative of the path, any sudden changes in the path height are detected by extracting the coordinates corresponding to peak values in the derivative. A second degree polynomial is fitted to the neighboring 40 pixels of the corresponding curve point $\left(x_{0}, y_{0}\right)$ to get a good estimate of the direction of the path around that peak. The result is applied to the original path,

$$
\begin{aligned}
y(x) & =y(x) w(x)+y^{\prime}(x)[1-w(x)] \text { for } \\
x & \in\left[x_{0}-M, x_{0}+M\right],
\end{aligned}
$$

where $y(x)$ is the original path, $y^{\prime}(x)$ is the fitted path, and $w(x)$ is a weight factor according to Eq. (10).

The pieces of the curve close to the tip-over moments obtain special treatment: a predefined number of curve points either to the left or right next to the tip-over moment is positioned according to the average increase in vertical position of the curve points before or after these points (respectively). Any tip-overs whose left and right paths have a small difference in height are probably not tip-overs and are therefore removed.

\section{Performance evaluation}

\section{a. Experiment}

To be able to test and evaluate our algorithm, the framework was tested on the data recorded in Beek $\left(50.92^{\circ} \mathrm{N}, 5.77^{\circ} \mathrm{E}\right)$, Netherlands, from 1954 to 1991 . Until 1979 daily strip charts were used, later recordings were done on rolls.

All strip charts and rolls were scanned, dated, and examined for artifacts and important annotations. The rolls were marked with day transitions using Roller. Each strip chart and every day on a roll was processed separately in a batch process. The results of the process were evaluated in PostACE. Finally, the results were converted with the aggregation program to 5-min time series of rainfall data.

\section{b. Results}

The overall detection quality of the ACE algorithm is good. In $70 \%$ of the total number of days processed the process was completed without erroneous results. If detection errors were noted during postprocessing, in most cases merely an adjustment of one or more of the found grid boundary locations was necessary. In special cases, usually during days with high-rainfall intensity, relocalization of the found curve path was sometimes necessary. Generally, the processing of a single day strip took $30-40 \mathrm{~s}$ on a Pentium 4 3.0-GHz machine. Depending on the presence and severity of the errors, postprocessing usually takes no longer than $1 \mathrm{~min}$, and on average it can be done within a fraction of that.

\section{1) General detection issues}

The most important reasons for failure of the automatic detection with ACE are listed in this section. In all cases the failures need to be corrected using PostACE.

\section{(i) Tip-over moments}

Normally, a pluviograph emptying its reservoir will result in an almost vertical line on the graph, which connects the recording lines just before and just after the tip-over moment. If this vertical line is clearly present on the paper, then it can easily be detected. However, in some cases the line is not or is hardly visible, and there is a chance that the algorithm misses the line and, therefore, the tip-over moment (Fig. 8a).

\section{(ii) Grid boundaries}

On many strips there are annotations or additional stamps visible outside of the grid. If these markings are of the same color as the grid itself, then the algorithm for detecting the grid boundaries might fail (Fig. 8b).

\section{(iii) Curve missed}

For similar reasons as above, the curve detection might fail and produce a curve passing through the annotations around the curve instead of the curve itself (Fig. 8c). Also, missed features like tip-overs may influence the outcome of the trace. In addition, in cases of either high or rapid changes in rainfall intensity small trace errors might arise during the steep change in height.

\section{(iv) Curve discontinuities}

The curve likelihood image displays the pixels that are likely to be part of the rainfall curve. Because of noise and discontinuities or gaps in the curve, this image might not contain one complete curve line, but several line parts belonging to that line. While small discontinuities of some consecutive pixels in the curve have negligible influence on the detection performance of the algorithm, larger gaps of several tens of pixels might influence the precision of the tracing around and during these gaps (Fig. 8d).

\section{(v) End points of the curve}

Because of holes in the curve and observer annotations the exact end points of the curve might be misdetected (Fig. 8e). Also, because of varying contrast across the image, parts of the curve might be misidentified. Therefore, the detected curve length might be shorter than visible on the strip chart. 
(a)

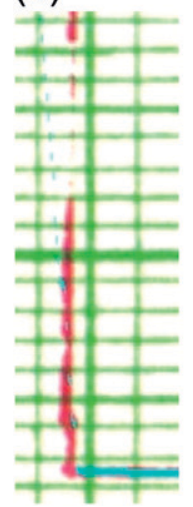

(b)



(c)

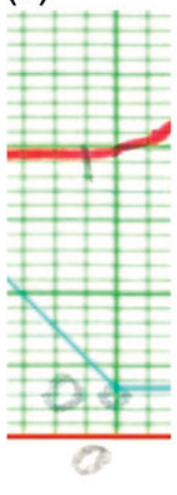

(d)

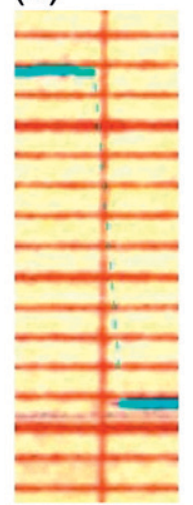

(e)

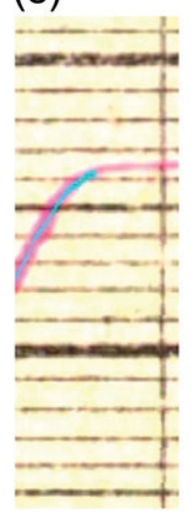

(f)

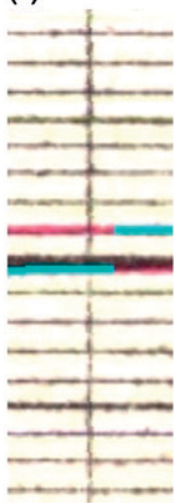

FIG. 8. Overview of typical failures arising during the detection process. In the images, all taken from different sources, the detected grid boundary positions (straight red lines) and the traced curve paths (turquoise lines) are shown. (a) The tip-over has not been detected. (b) The right boundary of the grid is not correctly located. (c) The path of the curve is incorrectly detected, probably because of the annotations below. (d) A hole in the curve causes the traced path to jump to the line above. (e) Misdetected curve end point. (f) Multiple lines on one strip chart cause sudden jumps in the traced path.

\section{(vi) Multiple lines on a single strip}

In various cases, multiple lines are visible on a single strip chart (Fig. 8f), meaning that multiple recordings have taken place over various consecutive days without replacing the strip chart after each day. This leads to tracing complications, such as jumps from one line to another in the resulting traced path. Currently the algorithm is only designed to trace one single path. To avoid the jumps and extract only a single line during postprocessing in PostACE, the redundant lines can be occluded. A retrace of the path will lead to correct results.

\section{2) Extreme value Distribution}

After postprocessing the data, the results have been aggregated to 5-min rainfall intensity values. In the case of Beek this yields a file with $36 \mathrm{yr}$ of 5-min data. These data can be used, for example, to perform extreme value analysis for different durations. As an example, Fig. 9 shows the Gumbel plots of the 10- and 60-min rainfall amounts of Beek. For each rainfall amount, the figure shows the corresponding return period $T_{r}$.

\section{Software framework}

The ACE software that we developed for rainfall chart images is freely available from the author. The framework consists of four documented programs. All of the programs use common code for the various overlapping functionalities. The code of the programs can be adjusted to suit the specific needs of each user.

The four programs are written in $\mathrm{C}++$. The GUI programs Roller and PostACE make use of the Microsoft
Foundation Classes (MFC) version 4.2 library included in Visual Studio 6. This framework originally required the purchase of a license, but because the actual code can be downloaded for free as part of the Windows Software Development Kit, this is no longer necessary. Because Visual Studio 6 is now generally regarded as outdated and poorly supports $\mathrm{C}++$ standards, the code has been updated to work with Visual C++ 2008 and 2010.

While some knowledge about image processing techniques is recommended for working with the applications, the design of the framework is such that one should be able to work intuitively while interacting with the Roller and PostACE programs. The chart processing and result aggregation tools are easy to use and can be configured

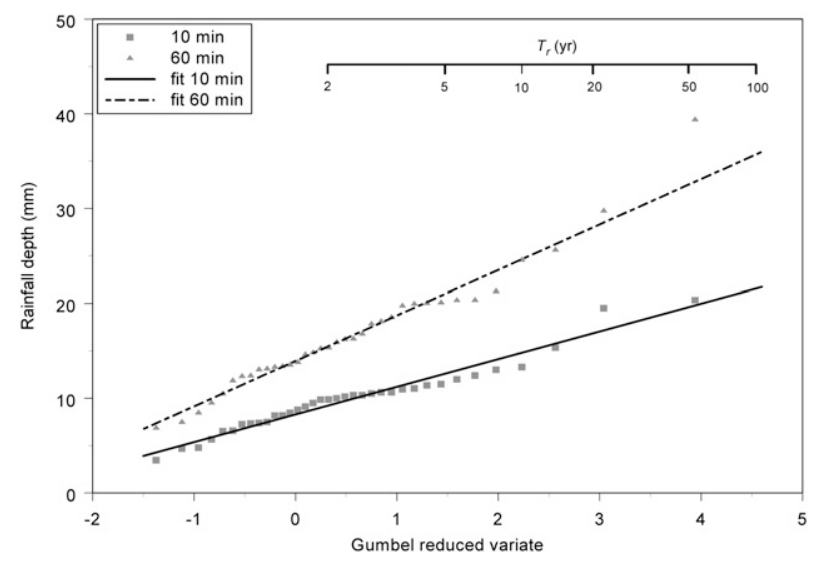

FIG. 9. Gumbel plots for the 10- and 60-min annual maximum rainfall amounts for Beek (1955-90). Maximum likelihood fits to the observed data are shown (solid and dashes lines). The annual maxima are plotted using the median plotting position. 
using command line parameters to quickly optimize detection results.

An extended document was written to instruct the user about how to run the programs and how to interact with the GUI applications. It also gives an overview about the program structures and various objects used throughout the framework.

\section{Discussion}

We developed the ACE algorithm for processing bulk quantities of rainfall strip charts and rolls. The idea was that many meteorological and hydrological institutes in the world possess large quantities of these hardcopy data in their archives and that these hardcopy data still contain information not yet available in digital form. ACE greatly reduces the amount of time for extracting this information, especially when high-resolution output is needed. Although the algorithm is developed for rainfall, it is possible to easily tailor it for other elements as well, as long as a clear chart with a curve on top of it is the main source of information. For instance, temperature, humidity, and air pressure have since long been recorded on similar charts. Also, if institutes are still operating with devices with strip charts for meteorological observation, then the ACE framework is easily applicable.

An important part of the work is the filling of a metadatabase. The database defines periods with identical features and contains information on the dates and times belonging to the curves on the charts and rolls and information contained in notes on the charts. Part of the work consists of checking handwritten notes on the charts. Because optical character recognition of this type of information is not yet feasible, this may take a considerable amount of time (depending on the type and amount of handwritten information).

The main advantage of ACE is that the strip chart processing algorithm does not need any prior knowledge about the color scheme of the chart. In Deidda et al. (2007) the results of processing depended on the predefined color ranges for each color channel. For each group of charts with a specific grid-curve color combination, these color ranges needed to be determined beforehand. We solved this issue by automatically analyzing the colors on the strip chart. Furthermore, by adapting the color model to each individual chart, ACE can achieve an accurate and robust result with unpredictable color variations, such as those caused by the variation of the thickness of the curve ink, the current state of the chart paper, and the (possibly) automatic color adjustments during the automatic scanning of charts.

ACE is suitable for the detection of only a single curve on a strip chart image. If multiple curves are present, then manual postprocessing is needed. Furthermore, the method assumes that the strip charts are positioned correctly onto the pluviograph during recording. Without this assumption there is no frame of reference and precise correction becomes problematic. For the charts and rolls processed here, there is no indication that strip charts were incorrectly positioned. For charts with curved vertical axes, we assumed that the curvature corresponds to the length of the arm of the pen. Correction of this curvature will then correctly reposition the rainfall curve line onto the now rectilinear grid. If the curvature of the grid does not correspond to the length of the arm of the pen, then there is no reliable way of correcting the curvature difference between the pen and the grid. In future developments, an extensive statistical analysis of the information extracted from the strip charts and their properties might give insight into the degree of error of the correction methods applied here. For example, for days for which it is known that there was no rain, the rainfall curves should be parallel to the horizontal grid lines. Analysis of this feature may provide an estimate of the systematic error in pluviograph chart positioning.

To ensure the correctness of the final result of each tracing, the result is visually checked by a user. Currently, there is no mathematical measure to determine whether detection and tracing were done properly. Although some checking is done during processing, error correction is far from complete. In future developments, such a measure might be useful, because the manual postprocessing and evaluation can then be limited to only the most extreme cases.

A possible improvement to the framework might be the use of the intermediate results of successive chart images as input for the color model of the next image to be processed. This will save a significant amount of time during the batch run of a number of images, because the color detection part could be omitted. This will, however, only be feasible when successive charts have the same color scheme in their images for extended periods of time. With the current version, the forced use of a color model of another image is already possible, but the intelligent application of color models among successive images has not been implemented.

\section{Conclusions}

An automatic curve extraction (ACE) procedure has been developed for digitizing large amounts of scanned rainfall strip charts and paper rolls. ACE uses a color detection procedure that automatically separates the background of the charts and rolls from the grid, and subsequently the rainfall curve. The rainfall curve is 
then detected by the minimization of a cost function. The procedure has successfully been applied to stations in the Netherlands resulting in $321 \mathrm{yr}$ of 5 -min rainfall sums. Manual postprocessing was needed to check the results of ACE. For this purpose a postprocessing tool has been developed that facilitates the correction of the results of $\mathrm{ACE}$, if needed.

Acknowledgments. The authors are grateful to Robert Leander and Henk van den Brink for their constructive advice during the early phase of this project. Roberto Deidda and two other anonymous reviewers are thanked for valuable comments. The work has been supported by the Dutch research program Climate Changes Spatial Planning.

\section{REFERENCES}

Alila, Y., 2000: Regional rainfall depth-duration-frequency equations for Canada. Water Resour. Res., 36, 1767-1778.

Bellman, R., 1957: Dynamic Programming. Princeton University Press, $336 \mathrm{pp}$

Burton, A., C. Kilsby, H. Fowler, P. Cowpertwait, and P. O'Connell, 2008: RainSim: A spatial-temporal stochastic rainfall modelling system. Environ. Modell. Software, 23, 1356-1369, doi:10.1016/ j.envsoft.2008.04.003.

Deidda, R., G. Mascaro, E. Piga, and G. Querzoli, 2007: An automatic system for rainfall signal recognition from tipping bucket gage strip charts. J. Hydrol., 333 (2-4), 400-412, doi:10.1016/j.jhydrol.2006.09.011.
Di Baldassarre, G., A. Castellarin, and A. Brath, 2006: Relationship between statistics of rainfall extremes and mean annual precipitation: An application for design-storm estimation in northern central Italy. Hydrol. Earth Syst. Sci., 10, 589-601.

Hogg, R., J. McKean, and A. Craig, 2005: Introduction to Mathematical Statistics. 6th ed. Pearson Prentice Hall, 692 pp.

Klein Tank, A. M. G., and G. Können, 2003: Trends in indices of daily temperature and precipitation extremes in Europe 194699. J. Climate, 16, 3665-3680.

— , and Coauthors, 2006: Changes in daily temperature and precipitation extremes in central and south Asia. J. Geophys. Res., 111, D16105, doi:10.1029/2005JD006316.

Lenderink, G., and E. V. Meijgaard, 2008: Increase in hourly precipitation extremes beyond expectations form temperature changes. Nat. Geosci., 11, 511-514.

Leonard, M., M. Lambert, A. V. Metcalfe, and P. S. P. Cowpertwait, 2008: A space-time Neyman-Scott rainfall model with defined storm extent. Water Resour. Res., 44, W09402, doi:10.1029/ 2007WR006110.

Madsen, H., P. Mikkelsen, D. Rosbjerg, and P. Harremoës, 2002: Regional estimation of rainfall intensity-duration-frequency curves using generalized least squares regression of partial duration series statistics. Water Resour. Res., 38, 1239, doi:10.1029/2001WR001125.

May, W., 2007: The simulation of the variability and extremes of daily precipitation over Europe by the HIRHAM regional climate model. Global Planet. Change, 57, 59-82.

Mikkelsen, P. S., H. Madsen, K. Arnbjerg-Nielsen, D. Rosbjerg, and P. Harremoës, 2005: Selection of regional historical rainfall time series as input to urban drainage simulations at ungauged locations. Atmos. Res., 77, 4-17.

Sonka, M., V. Hlavac, and R. Boyle, 1998: Image Processing, Analysis, and Machine Vision. 3d ed. CL-Engineering, 800 pp. 